\title{
Optimization Data Fusion Approaches for Intelligent House System
}

\author{
Wen-Tsai Sung ${ }^{1}$, Jui-Ho Chen ${ }^{2}$ and Hsi-Chun Wang ${ }^{3}$ \\ ${ }^{1}$ Department of Electrical Engineering, National Chin-Yi University of Technology, Taiwan. No.57, Sec. 2, Zhongshan Rd., Taiping \\ Dist., Taichung 41170, Taiwan. \\ ${ }^{2}$ Department of Electrical Engineering, National Chin-Yi University of Technology, Taiwan. No.57, Sec. 2, Zhongshan Rd., Taiping \\ Dist., Taichung 41170, Taiwan. \\ ${ }^{3}$ Department of Electrical Engineering, National Chin-Yi University of Technology, Taiwan. No.57, Sec. 2, Zhongshan Rd., Taiping \\ Dist., Taichung 41170, Taiwan.
}

Received: 25 Oct. 2012, Revised: 22 Jan. 2013, Accepted: 25 Jan. 2013

Published online: 1 Jun. 2013

\begin{abstract}
This study production of smart house to show the entire system, the use of self-sensing module and the 8051 monocrystal chip processing sensor signals, the success achieved with the measurement of temperature, humidity, illumination, carbon monoxide sensor module. This study purposes the smart house, remote monitoring and automatic system based on consistency algorithm for multi-sensor optimization data fusion via wireless sensors network framework. The way transmits by wireless (ZigBee chips) and wired (DAQ signal acquisition device) sensor value send to the server-side carries on remote monitoring and record etc. In accordance with environmental requirements set in the server-side sensing value, using the infrared ray way automatic control load. When it necessity also can use the available hand-hold remote control, dual control functions to enable the environment to be safer and the comfortable home life.
\end{abstract}

Keywords: Intelligent environmental monitoring system, ZigBee wireless technology, infrared control, automatic control.

\section{Introduction}

As technology advances, the humanity lives the quality improvement, information technology and the wisdom life has become the inevitable trend. Smart home is in response to the needs of the times, also will be future home new state. [1] In addition most important carries out the goal is the life security safeguard, the comfort of home, disaster prevention, emergency and other integrated into the health, safety management systems. Through user-friendly operation interface, reducing the safety hazards at home, the promotion occupies the environment comfort level, safeguard home life security.[2]

Many companies are already actively involved in industry and tertiary institutions to the development of smart home systems, such as the Taiwan Institute of Technology and Building Research Institute Ministry of the Interior together to create the Green House model, [3] Schneider Electric launched Wiser intelligent home control etc., [4] thus it can be seen the wisdom home system receives takes seriously. Because of this, this study aims to develop intelligent real-time environmental monitoring and home automation systems, and Smart house to render. The way transmits by wireless (ZigBee chips) and wired (DAQ signal acquisition device) sensor value send to the server-side carries on remote monitoring and record etc. Finally use infrared control load, so as to reduce its cost and the lift system stability, achieves the so-called wisdom control.

\section{System hardware architecture}

Using the self-made sensing mold train, the physical signals into electrical signals, and do linear process, and through A / D converted into digital signals. Using 8051 monocrystal chips digital signal cumulative collection and then take the average of the values shown in the seven-segment display, while taking advantage of ZigBee

* Corresponding author e-mail: songchen@ ncut.edu.tw 
chips receive data transmission packets to the Gateway, and then use Ethernet transmitted to server-side for remote monitoring. Another, Environmental sensor can be directly done by the DAQ signal acquisition device to do wire transfer. In the server-side man-machine interface, the wireless monitoring is designs by Visual Basic6.0, wired are by the LabVIEW to design, the entire system architecture shown in Fig.1. The load is automatically controlled by Infrared ray to adjust the environment, when necessity also can use the available hand-hold remote control, and presents whole set system by the smart house.

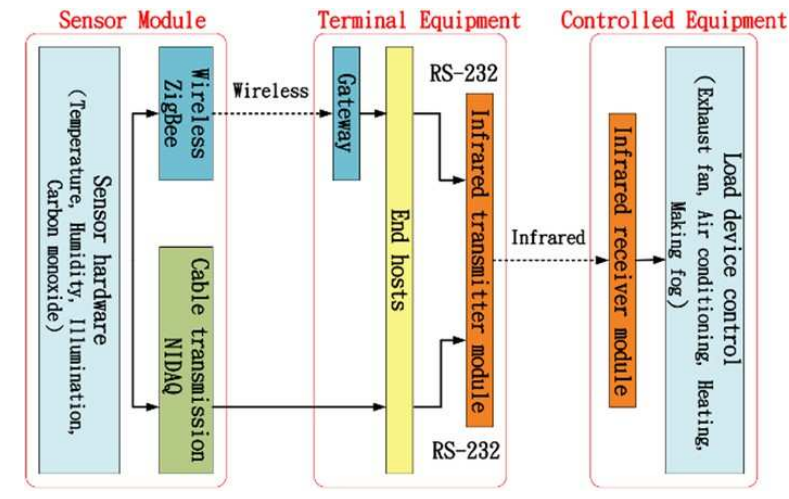

Fig. 1 System architecture diagram

\section{Consistency data fusion algorithm}

According to sung; [4] the method of multi-sensor measurement data consistency fusion, first of all, the definition of confidence distance measurement which is a kind of measurement that balance the support level of odometer measurement data, is established between confidence distance matrix and relation matrix in basic structure of the multi-odometer measurement data. By utilizing digraph method, a plenty of inaccuracies or errors can be deleted or removed. Finally, this paper adopts the maximum likelihood estimation (MLE) to make the best fusion value of multi-odometer measurement data.

When this study employed multi-sensor to measure the same parametric, this investigate can assume that two sensors $(i, j)$ are measurable values $\left(X_{i}, X_{j}\right)$ which are following the Gaussion distribution. Each pdf curve line from measurable value is used to make characteristic function $\left(\mathrm{p}_{\mathrm{i}}(\mathrm{x}), \mathrm{p}_{\mathrm{j}}(\mathrm{x})\right)$. We record the first measurable values $\left(\mathrm{X}_{\mathrm{i}}, \mathrm{X}_{\mathrm{j}}\right)$ are $\mathrm{x}_{\mathrm{i}}, \mathrm{x}_{\mathrm{j}}$. To make the deviations between $\mathrm{x}_{\mathrm{i}}$ and $\mathrm{x}_{\mathrm{j}}$, we adopt conception of confidence distance measurement as following,[5]

$$
\begin{gathered}
d_{i j}=2 \int_{x i}^{x} p_{i}\left(x \mid x_{i}\right) d x=2 A \\
d_{j i}=2 \int_{x j}^{x} p_{j}\left(x \mid x_{i}\right) d x=2 B \\
p_{i}\left(x \mid x_{i}\right)=\frac{1}{\sqrt{2 \pi} \sigma_{i}} \exp \left[-\frac{1}{2}\left(\frac{x-x_{i}}{\sigma_{i}}\right)^{2}\right] \\
p_{j}\left(x \mid x_{j}\right) d x=\frac{1}{\sqrt{2 \pi} \sigma_{j}} \exp \left[-\frac{1}{2}\left(\frac{x-x_{j}}{\sigma_{j}}\right)^{2}\right]
\end{gathered}
$$

The confidence distance measurement from sensors ( $\mathrm{i}, \mathrm{j})$ are called $\left(d_{i j}, d_{j i}\right)$, both reflect measurement values with consistency. The value from $d_{i j}$ is lower, observation from both get similar, otherwise, the deviation gets higher. Therefore, the fusion from both sensors is called $d_{i j}$. A, B is probability density curve square measure $p_{i}\left(x \mid x_{i}\right), p_{j}\left(x \mid x_{j}\right)$ on internal $\left(x_{i}, x_{j}\right)$.

$$
\begin{gathered}
\text { When } x_{i}=x_{j} \text {, then } d_{i j}=d_{j i}=0 . \\
\text { When } x_{i}>x_{j} \text { or } x_{i}<x_{j} \text {, then } d_{i j}=d_{j i}=1 .
\end{gathered}
$$

If the (m) sensor, which measured the same parameter, then confidence distance measurement $d_{i j}(i, j=1,2, \cdots, m)$ which established the matrix of $D_{m}$, called multi-sensor data confidence distance matrix which describes each sensor support level with consistency.[6] Data combination sensor is the best fusion data, which is used to make a result from Measured Parameters. We adapt one data from all fusion value that probability density function as following in an equation (5) and Maximum Likelihood function in function (6)

$$
\begin{gathered}
p_{i}\left(x_{i} \mid \theta\right)=\frac{1}{\sqrt{2 \pi} \sigma_{i}} \exp \left[-\frac{1}{2}\left(\frac{x_{i}-\theta}{\sigma_{i}}\right)^{2}\right] \\
v i=1,2, \ldots ., l \\
L\left(x_{1}, x_{2}, \ldots, x_{l} ; \theta\right)=\prod_{i=l}^{l} p_{i}\left(x_{i} \mid \theta\right)
\end{gathered}
$$

this investigate can get the best fusion value form original measurement data $\widehat{\theta}=\widehat{\theta}\left(x_{1}, x_{2}, \ldots, x_{l},\right)$ which should satisfied as following equations:

$$
L\left(x_{1}, x_{2}, \ldots, x_{l} ; \theta\right)=\sup _{\theta \in \Theta} L\left(x_{1}, x_{2}, \ldots, x_{l} ; \theta\right)
$$

According to the equation (7), we can get natural logarithms for both sides, then the equation as following (8)

$$
\ln L\left(x_{1}, x_{2}, \ldots, x_{l} ; \theta\right)=\sup _{\theta \in \Theta} \ln L\left(x_{1}, x_{2}, \ldots, x_{l} ; \theta\right)
$$


According to the purpose of the maximum likelihood estimation, we can get $\left.\frac{\partial}{\partial \theta} L\left(x_{1}, x_{2}, \ldots, x_{l} ; \theta\right)\right|_{\theta=\widehat{\theta}}=0$ which is equal to

$$
\frac{\partial}{\partial \theta} \sum_{i=1}^{l} \ln \frac{1}{\sqrt{2 \pi} \sigma_{i}} \exp \left[-\frac{1}{2}\left(\frac{x_{i}-\theta}{\sigma_{i}}\right)^{2}\right]_{\theta=\widehat{\theta}}=0
$$

this investigate got the solution as following,

$$
\widehat{\theta}=\frac{\sum_{i=1}^{l} \frac{x_{i}}{\sigma_{i}}}{\sum_{i=1}^{l} \frac{1}{\sigma_{i}}}
$$

$\widehat{\theta}$ is fusion set $\left\{x_{1}, x_{2}, \ldots, x_{l} ;\right\}$ the best fusion data.[7]

\section{Experimental results}

Sensors

This study production of sensors has temperature (AD590), humidity (C2-M3), degree of illumination (Cds), the carbon monoxide (TGS800), shown in figure 2

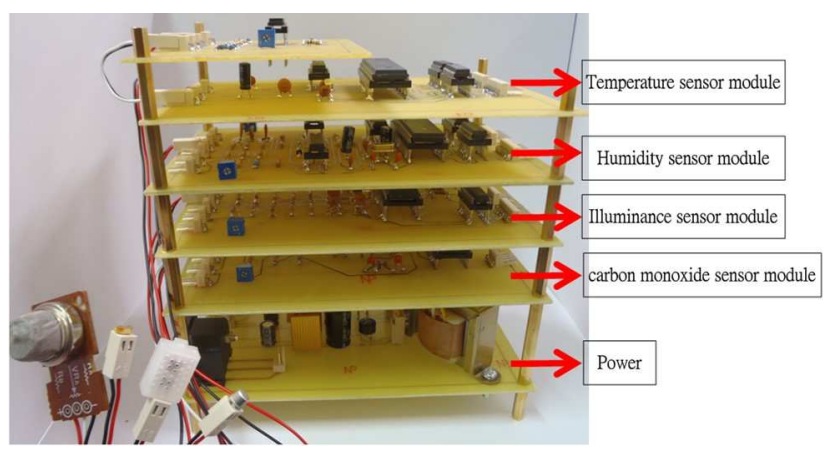

Fig. 2 The sensor module

\section{Infrared control load}

Infrared is by PT2248, PT2249 and 8051 manufactures, has the transmitting end and the receiving end, shown in figure 3(a)(b). A server-side order is to use RS-232 sent to the infrared transmitter to determine, coding, and then by the infrared receiver to, decode, determine signal needed to control the load. In this study control's load has the air conditioners and dehumidifiers, heating, fog devices, exhaust fan, three sections of lighting, shown in figure 4 (a) ${ }^{\sim}(d) .[8]$
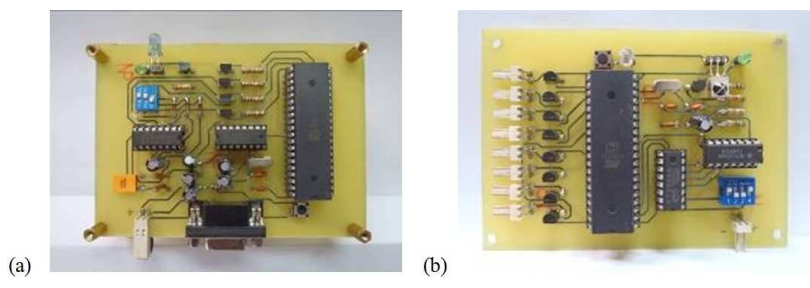

Fig. 3 (a) Infrared transmitter module (b) Infrared receiver module
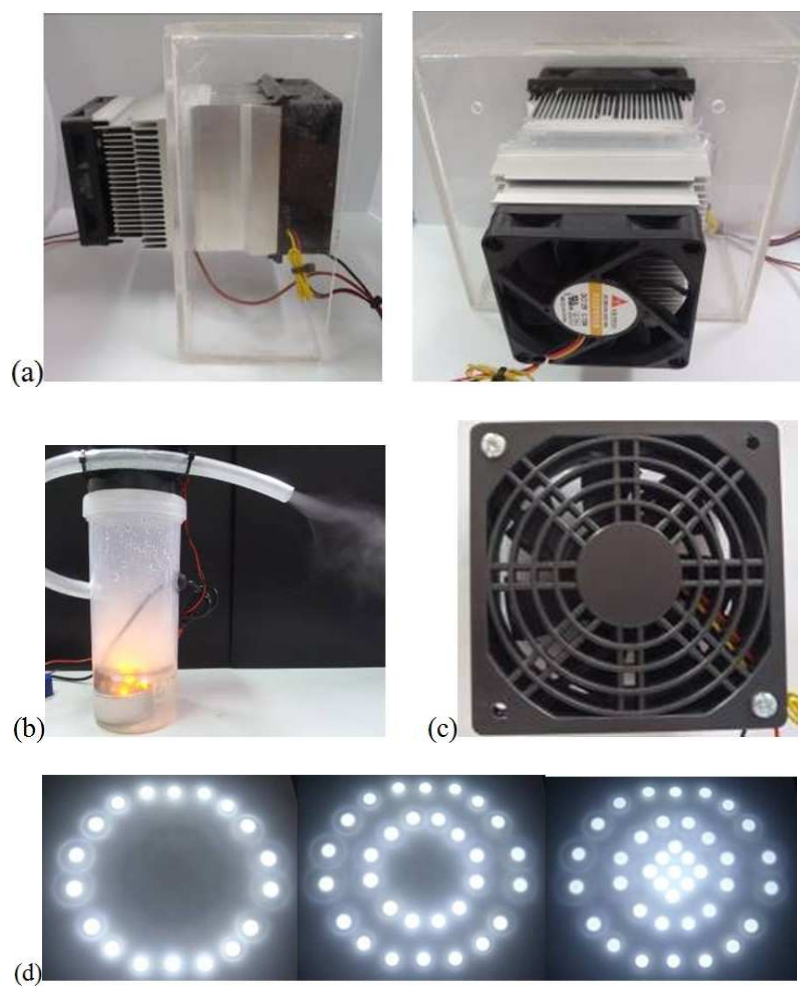

Fig. 4 (a) Air conditioners and dehumidifiers, heating load (b) Fog devices (c)Exhaust fan(d)Three sections of lighting

\section{Wireless automatic monitoring system interface}

The wireless monitoring divides into three interfaces: the current environment, load control, abnormal records, additionally records can stores up in Excel, shown in figure 5 (a) (d) [9]

When automatic control, in order to reduce the load of the switch, so when the temperature sensor value is equal to " setting value \pm 1 ", the state is normal, without any action; Larger than " setting value +1 ", the state is too high, it will save the abnormal, and open the air conditioning, when the value is less than " setting value -1 "will be closed; And less than or equal " setting value 


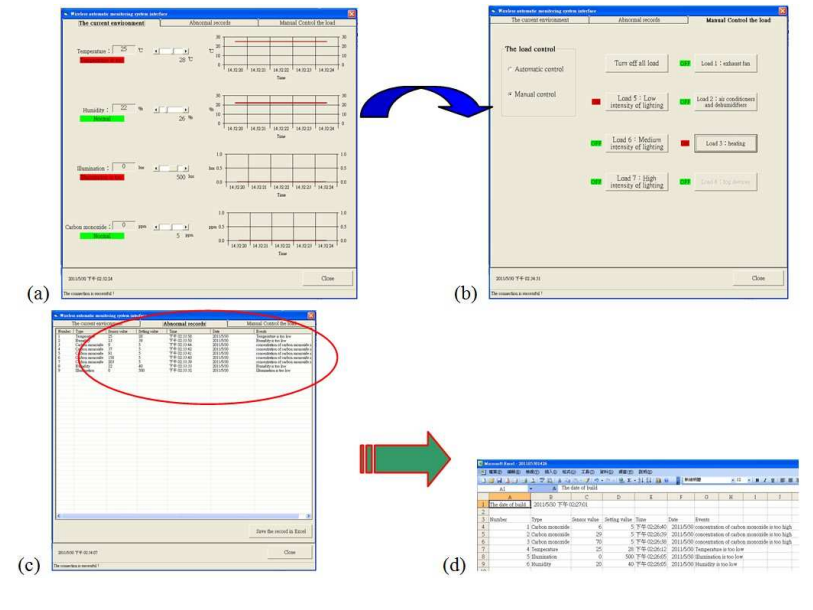

Fig. 5 (a) The current environment (b) Load control(c) Abnormal records (d) Recorded in Excel

-3 ", the state is too low, and opens the heating until the sensor is equal to" setting value".
The humidity sensor value is equal to " setting value $\pm 5 \%$, the state is normal, without any action; Larger than "setting value +5 ", state is too high, and opens the dehumidifier until the sensor value is equal to "setting value -5 ", and the state is normal; Less than " setting value -7", the state is too low, and opens fog devices until the sensor value is equal to "setting value", and the state is normal;

The degree of illumination setting value is 0 , sensor value may in $0 \sim 150$; setting value is 300 , sensor value may in 400 600; setting value is 700, sensor value may in 600 800; Status is normal, without any action; When the sensor value is not in the normal range, larger than the status is too high, less than the status is too low, and opens the illumination which needs. Carbon monoxide sensor value is larger than setting value, the status is too high, and opens the exhausts fan to remove the carbon monoxide.

When Manual control, can easily switch the load moves, but the load has priority of the points, when the carbon monoxide is too high and the temperature overheated synchronize. The first dealing with the problem of high carbon monoxide, and opens the exhaust fan, when the state is normal, continue to address the overheating temperature, and so sorting is the exhaust fan, air conditioners and dehumidifiers, heating, fog devices, and lighting can be in accordance with according to personal settings.

When the smart house is automatic control, the environment sensor value is unusual, will open the required load to achieve the immediate monitoring. If two or more kind of above sensing values are simultaneously unusual, the load from the open top priority and the status back to normal, then open the next load.

\section{Wired automatic monitoring system interface}

The wired monitoring divide into five interfaces: Wired automatic monitoring interface, temperature, humidity, illumination, carbon monoxide monitoring interface, additionally information is also stored in Excel, as shown in figure 6 to 7 . In the Internet world, the monitoring interface can be opened directly from the computer's IE, in any place immediate monitoring sensing gauging data, as shown in figure 8 .
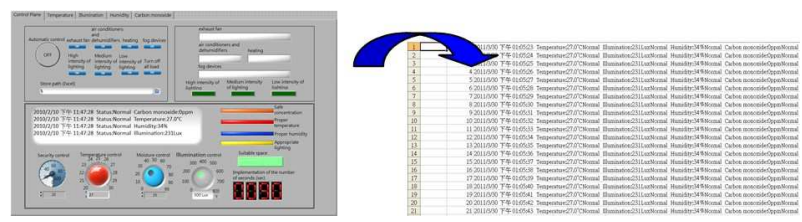

Fig. 6 Wired automatic monitoring interface and data stored in the Excel 
(a)
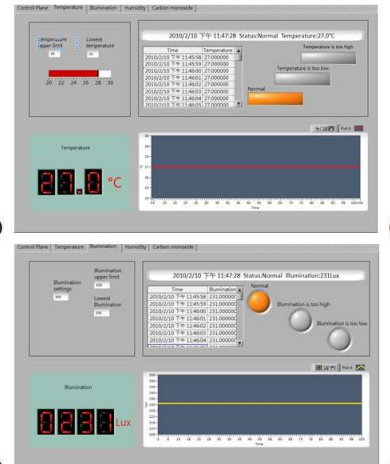

(b)

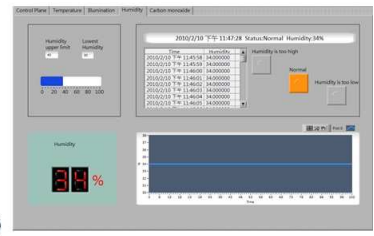

(c)

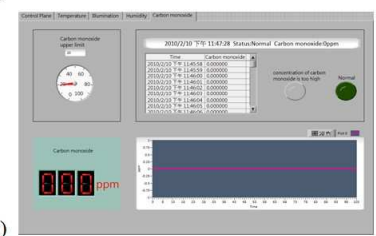

Fig. 7 (a) Temperature interface (b) Humidity interface (c) Illumination interface (d) Carbon monoxide interface

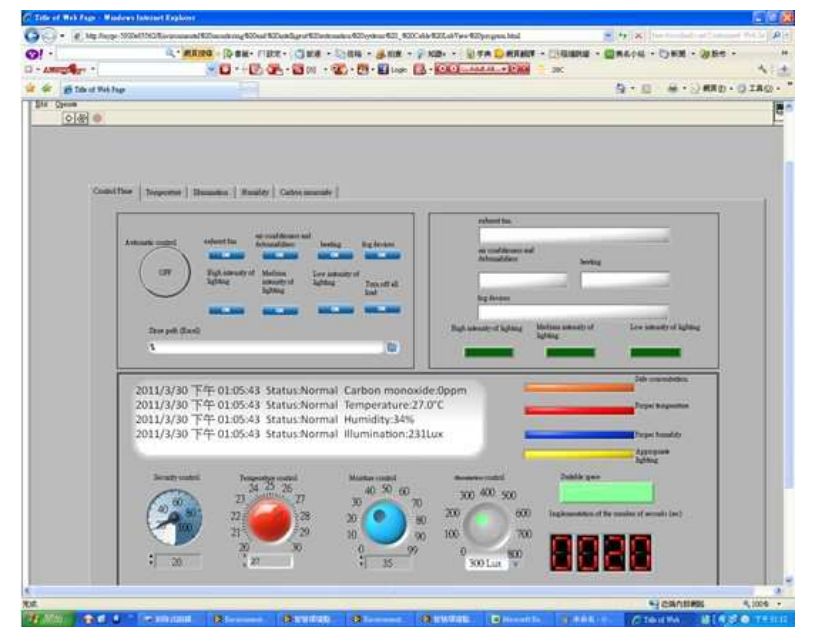

Fig. 8 IE monitoring interface

In the automatic control, the program will be based on the current state of the environment to open the corresponding load, is similar to the wireless automation supervisory system interface, manual control, too.

\section{Smart house}

This study production of smart house to show the entire system, as shown in figure 9, and the location of the sensors shown in figure 10.

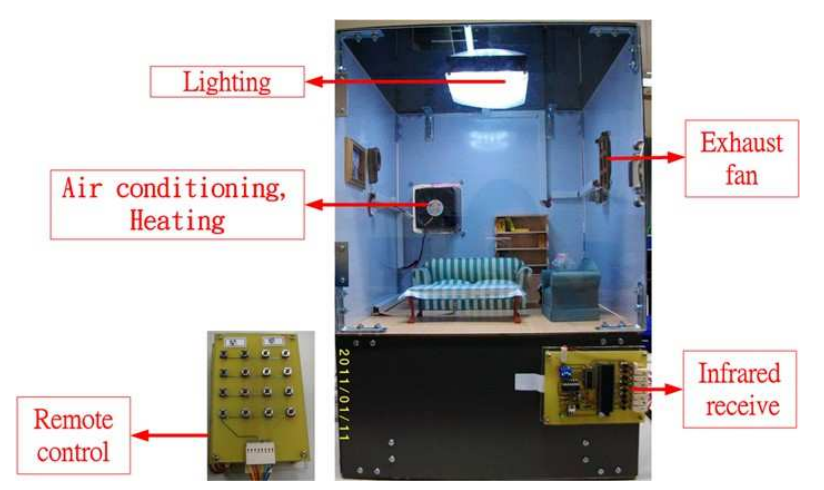

Fig. 9 Smart house

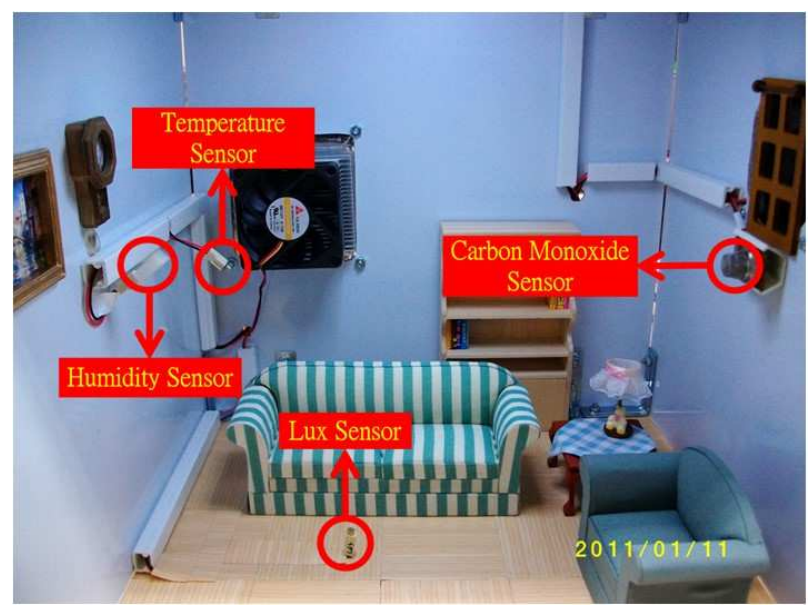

Fig. 10 The sensor location 
The result of Sensor data fusion and implement standard of adapting maximum likelihood algorithm and average algorithm is shown as figure 7 and figure 8 . This investigate can got some suggestion from the analysis figure with curve, to delete or remove the extremely values or the values with errors, this investigate can adapts maximum likelihood algorithm with confidence data to make our research more accuracy. However, to measure the data with nearly, the result of data fusion algorithm is better than average fusion algorithm.

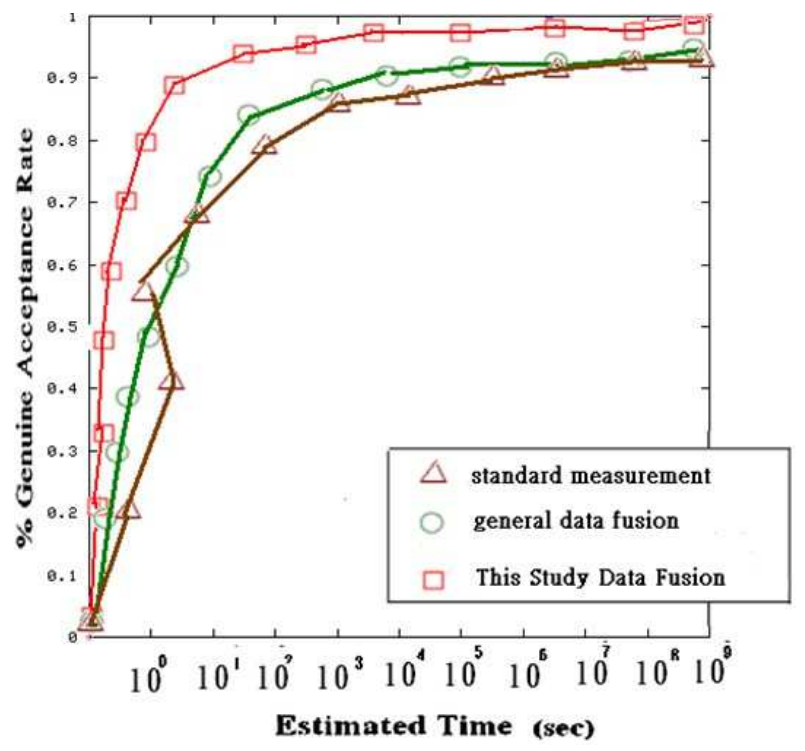

Fig. 11 Results of traditional data fusion methods compare with innovative optimization data fusion approaches.

In figure 11, when the estimated time approached $10^{9}$ sec above the actual value, the measurement value tended to match. The experiment compared the optimization data fusion approaches based; standard measurement and general data fusion methods based on parallel data fusion methods. The standard measurement based cited the literature from our pervious researches in the [10] reference list. According to the training error curves, the optimization data fusion approaches based method was better than the other methods in measurement process.

\section{Conclusion and Future Work}

Most of the work required to operate under a particular environmental, this study combined with other fields to develop the conditions necessary for its working environment control, to reduce the impact of environmental change on the work to achieve high stability, high efficiency and high accuracy Work environment and results.
In the future we hope to develop low-cost, low energy consumption, energy saving control, wisdom, and human intelligent environmental control system. Then combined with the sustainable use of recyclable building materials, electric power energy completely self-sufficient, through various the rapid development of Internet developed a more perfect environment monitoring system.

\section{Acknowledgement}

This research was supported by the National Science Council of Taiwan under grant NSC 100-2218-E-167-001. The authors would like to thank the National Chin-Yi University of Technology, Taiwan for financially supporting this research.

\section{References}

[1] Yu-Wei Chen, "Monitoring and Control of Home Environment Using Web-Based Wireless Network", National Taiwan University Department of Bio-lndustrial Mechatronics Engineering, 2007.

[2] Ya-Ling Huang, "Interface Design for Security System of Smart Home", National Taipei University of Technology Industrial Design, 2003.

[3] Min-Jung $\mathrm{Hu}$, "Green Smart house - Province 4 into electricity", Apple Daily, December 19, 2009.

[4] Wen-Tsai Sung, Yao-Chi Hsu, "Designing an Industrial Real-time Measurement and Monitoring System Based on Embedded System and ZigBee ", Expert Systems with Applications, Elsevier Company. Vol. 38, Issue 4, pp. 45224529. April 2011.

[5] Jey, "Schneider Electric launched Wiser "smart home" control system", June 8, 2010.

[6] M. Cardei, M. T. Thai, Y. Li, and W. Wu, "Energy-efficient target coverage in wireless sensor networks," Proc. IEEE INFOCOM, pp. 19761984, 2005.

[7] T. C. Shermer, "Recent results in art galleries," Proc. IEEE, vol. 80, no. 9, pp. 13841399, Sep. 1992.

[8] Khusvinder Gill, Shuang-Hua Yang, Fang Yao, and Xin Lu, "A ZigBee-Based Home Automation System", IEEE Transactions on Consumer Electronics, Vol. 55, No. 2, MAY 2009.

[9] Chia-Yu Tu, "A Study of Sensor-based ZigBee Technology for Healthcare Application", Department of Information Engineering, Asia University, 2005.

[10] Wen-Tsai Sung, Hung-Yuan Chung, Yao-Chi Hsu, KuanYu Chen, "A Light Wind Speed Measurement System Design to Strengthen Wireless Sensor Network Functions", International Journal of Innovative Computing, Information and Control, Vol.7, No.2, pp.859-868. Feb. 2011.

Wen-Tsai Sung is working with the Department of Electrical Engineering, National Chin-Yi University of Technology as a professor. He received a PhD and MS degree from the Department of Electrical Engineering, 
National Central University, Taiwan in 2007 and 2000. He has won the 2009 JMBE Best Annual Excellent Paper Award and the dragon thesis award that sponsor is Acer Foundation. His research interests include Wireless Sensors Network, Data Fusion, System Biology, System on Chip, Computer-Aided Design for Learning, Bioinformatics, and Biomedical Engineering. He has published a number of international journal and conferences article related to these areas.

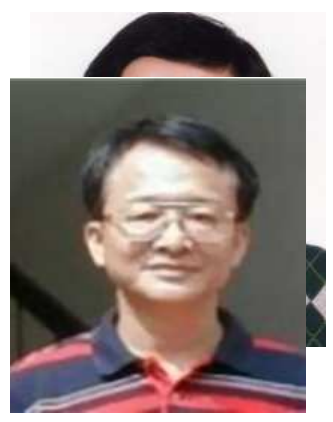

Jui-Ho Chen is working with the Department of Electrical Engineering, National Chin-Yi University of Technology as a professor. His research interests include Wireless Sensors Network, Data Fusion, and Biomedical Engineering. $\mathrm{He}$ has published a number of international journal and conferences article related to these areas.

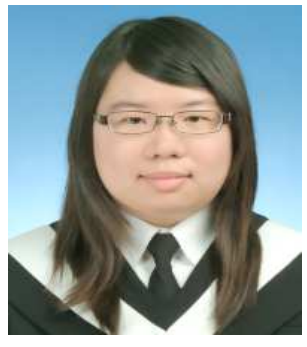

Networks Laboratory.

\section{Hsi-Chun}

Wang is a master student at the Department of Electrical Engineering, National Chin-Yi University of Technology. His Current research activities include the Wireless Sensors Networks, SoC System and Data fusion, etc. Currently, he is the member of Wireless Sensors 